\title{
Fluxos de óxido nitroso e suas relações com atributos físicos e químicos do solo
}

\author{
Rubia Santos Corrêa(1), Beáta Emöke Madari(2), Glaucilene Duarte Carvalho(3), \\ Adriana Rodolfo da Costa $^{(4)}$, Ana Cláudia de Castro Pereira ${ }^{(1)}$ e João Carlos Medeiros ${ }^{(5)}$
}

\begin{abstract}
(1)Universidade Federal de Goiás, Escola de Agronomia, Caixa Postal 131, CEP 74001-970 Goiânia, GO, Brasil. E-mail: rubiascorreagyn@gmail.com, anaclaudia_castro2@hotmail.com (2)Embrapa Arroz e Feijão, Rodovia GO-462, Km12, Caixa Postal 179, CEP 75375-000 Santo Antônio de Goiás, GO, Brasil. E-mail: beata.madari@embrapa.br ${ }^{(3)}$ Secretaria de Meio Ambiente e Recursos Hídricos, 11aㅗ Avenida, no 1.272, Setor Leste Universitário, CEP 74605-060 Goiânia, GO, Brasil. E-mail: glaucilene_agro@yahoo.com.br (4)Universidade Estadual de Goiás, Campus Santa Helena de Goiás, Via Protestato Joaquim Bueno, no 945, Vila Santa Helena, CEP $75920-000$ Santa Helena, GO, Brasil. E-mail: adriana.costa@ueg.br ${ }^{(5)}$ Universidade Federal do Piauí, Campus Professora Cinobelina Elvas, Estrada Municipal Bom Jesus-Viana, Planalto Horizonte, CEP 64900-000 Bom Jesus, PI, Brasil. E-mail: medeiros.jc@gmail.com
\end{abstract}

Resumo - O objetivo deste trabalho foi avaliar a dinâmica dos fluxos de óxido nitroso $\left(\mathrm{N}_{2} \mathrm{O}\right)$ em solo sob pastagem, em sistema de integração lavoura-pecuária, e sob cerrado nativo usado como referência, bem como identificar os atributos físicos e químicos do solo que influenciam esses fluxos. O estudo foi realizado na Fazenda Capivara, da Embrapa Arroz e Feijão, de 5 de fevereiro a 30 de setembro de 2013. As amostragens de gás foram realizadas com uso de câmaras estáticas manuais. O solo sob pastagem apresentou os maiores fluxos de $\mathrm{N}_{2} \mathrm{O}$, com média de $14,12 \mathrm{ng} \mathrm{m}^{-2} \mathrm{~s}^{-1}$. Essa área também apresentou maior espaço poroso preenchido por água (EPPA), que, associado à temperatura e ao teor de nitrato no solo, influenciou a produção de $\mathrm{N}_{2} \mathrm{O}$. $\mathrm{Na}$ época chuvosa, os fluxos apresentaram correlação positiva com o EPPA do solo e, na época seca, com seu teor de amônio e nitrato. A emissão total de $\mathrm{N}_{2} \mathrm{O}$ na área da pastagem foi de $1.644,19 \mathrm{~g}$ ha ${ }^{1}$. No cerrado nativo, não houve emissão de $\mathrm{N}_{2} \mathrm{O}$ do solo para a atmosfera.

Termos para indexação: Urochloa, desnitrificação, espaço poroso preenchido por água, gás de efeito estufa, nitrificação.

\section{Nitrous oxide fluxes and their correlations with physical and chemical soil properties}

\begin{abstract}
The objective of this work was to evaluate the dynamics of nitrous oxide $\left(\mathrm{N}_{2} \mathrm{O}\right)$ fluxes in a soil under pasture, in an integrated crop-livestock system, and in native cerrado as a reference, besides identifying the physical and chemical soil properties that affect the fluxes. The study was conducted at Fazenda Capivara of Embrapa Arroz e Feijão, in the state of Goiás, Brazil, between February 5 and September 30, 2013. Gas sampling was performed using manual static chambers. The soil under pasture showed the highest $\mathrm{N}_{2} \mathrm{O}$ fluxes with average of $14.12 \mathrm{ng} \mathrm{m}^{-2} \mathrm{~s}^{-1}$. This area also showed greater water-filled pore space (WFPS), which, associated with soil temperature and nitrate content in the soil affected the production of $\mathrm{N}_{2} \mathrm{O}$. In the rainy season, fluxes showed positive correlation with soil WFPS, and, in the dry season, they were correlated with soil contents of ammonium and nitrate. The total $\mathrm{N}_{2} \mathrm{O}$ emission in the pasture area was $1,644.19 \mathrm{~g} \mathrm{ha}^{-1}$. In native cerrado, there was no soil- $\mathrm{N}_{2} \mathrm{O}$ emission to the atmosphere.
\end{abstract}

Index terms: Urochloa, denitrification, water-filled pore space, greenhouse gas, nitrification.

\section{Introdução}

O clima da Terra é regulado pela energia solar que atravessa a atmosfera na forma de luz visível. Parte dessa energia é absorvida pela superfície terrestre, e parte é refletida de volta ao espaço na forma de radiação infravermelha. Determinados gases que compõem a atmosfera terrestre - dentre os quais se destacam o dióxido de carbono $\left(\mathrm{CO}_{2}\right)$, o metano $\left(\mathrm{CH}_{4}\right)$, o óxido nitroso $\left(\mathrm{N}_{2} \mathrm{O}\right)$ e o vapor d'água - absorvem parte das radiações infravermelhas emitidas pela superfície da Terra e pela atmosfera, dificultando sua dissipação para o espaço. Estes gases, conhecidos como gases de efeito estufa (GEE), fazem com que a temperatura média do planeta se situe ao redor de $14^{\circ} \mathrm{C}$, o que é importante para o surgimento e a manutenção de vida, pois, sem eles, a temperatura à superfície da Terra seria $33^{\circ} \mathrm{C}$ mais fria (Machado, 2005). 
Entretanto, aumentos recentes das concentrações dos GEE na atmosfera, em parte associados a atividades antrópicas, têm causado impacto sobre o balanço de radiação do planeta e sobre a temperatura da superfície da Terra. Entre os gases que absorvem a radiação infravermelha, o $\mathrm{N}_{2} \mathrm{O}$ é um importante GEE pois, apesar de sua baixa concentração na atmosfera, ele se destaca pelo longo tempo de permanência e pelo alto potencial de aquecimento global (PAG). O PAG da molécula de $\mathrm{N}_{2} \mathrm{O}$ é 290 vezes superior ao da de $\mathrm{CO}_{2}$, para um período de 20 anos, e 330 vezes superior, para um período de 100 anos (Brasseur et al., 1999).

No Brasil, em 2010, 35\% das emissões nacionais de GEE foram provenientes da agropecuária, e $95,44 \%$ das emissões de $\mathrm{N}_{2} \mathrm{O}$ oriundas desse setor são provenientes dos solos agrícolas; ou seja, do esterco dos animais em pastagem, do uso de fertilizantes sintéticos nitrogenados, da aplicação de adubos orgânicos, da incorporação de resíduos agrícolas ao solo, das áreas de cultivo de solos orgânicos e de emissões indiretas. Os animais em pastagem destacam-se quanto às emissões, com 44\% das emissões diretas (Estimativas..., 2013).

As atividades agrícolas que influenciam a emissão de $\mathrm{N}_{2} \mathrm{O}$ do solo para a atmosfera é mediada por microrganismos, e os processos de nitrificação e desnitrificação são os mais importantes. Entre os fatores que influenciam esses processos, destacam-se a umidade, as temperaturas do ar e do solo, o $\mathrm{pH}$, a textura, o manejo do solo e a rotação de culturas (Snyder et al., 2009).

$\mathrm{O}$ uso e o manejo conservacionista do solo pela adoção de sistemas integrados associados a práticas que empregam o revolvimento mínimo do solo, a cobertura da superfície do solo e a rotação de culturas podem favorecer a alta produção de resíduos vegetais e o acúmulo de nutrientes na superfície do solo e, portanto, aumentar os estoques de $\mathrm{C}$ do solo contribuindo para mitigar a emissão de GEE (Lal et al., 2007). No Brasil, em regiões como no Cerrado, a adoção de práticas de manejo conservacionista, a exemplo do sistema de plantio direto, contribui para o sequestro de 9 megatonelada de C por ano (Cerri \& Cerri, 2007).

No Cerrado, a integração lavoura-pecuária (ILP) vem-se expandindo com maior velocidade. $\mathrm{O}$ sistema ILP é uma alternativa para reverter a degradação das pastagens e melhorar a qualidade do solo e seu teor de matéria orgânica (Vilela et al., 2012). No potencial de sinergismo entre os componentes pastagem e lavoura encontra-se grande parte dos efeitos benéficos da ILP (Alvarenga \& Noce, 2005), entre os quais podem ser citados: a melhoria das propriedades físicas, químicas e biológicas do solo; o potencial de redução de riscos econômicos, pela diversificação de atividades; a redução do custo na recuperação/renovação de pastagens em processo de degradação; o aumento da matéria orgânica do solo e, consequentemente, menor perda por escorrimento superficial; o sequestro de carbono; e a mitigação das emissões de GEE (Vilela et al., 2012).

O objetivo do presente estudo foi avaliar a dinâmica dos fluxos de óxido nitroso $\left(\mathrm{N}_{2} \mathrm{O}\right)$ em solo sob pastagem, em sistema de integração lavoura-pecuária, e em um fragmento de cerrado nativo usado como referência, bem como identificar os atributos físicos e químicos do solo que influenciam esses fluxos.

\section{Material e Métodos}

O presente estudo foi desenvolvido na área experimental da Embrapa Arroz e Feijão, na Fazenda Capivara, no Município de Santo Antônio de Goiás (16²9'59"S, 49¹7'35"W), GO, na região Centro-Oeste do Brasil. A altitude média da área é de $801 \mathrm{~m}$, e a declividade é de aproximadamente $0,3 \%$. O solo é um Latossolo Vermelho acriférrico típico, de textura argilosa, com $530 \mathrm{~g} \mathrm{~kg}^{-1}$ de argila (Santos et al., 2010). O cerrado nativo, fase cerradão, de aproximadamente 216 ha, cujo fragmento foi adotado como área de referência, apresenta vegetação subcaducifólia. O clima regional é o tropical de savana megatérmico, tipo Aw segundo a classificação de Köppen-Geiger, marcado por duas estações - chuvosa, de outubro a abril, e seca, de maio a setembro. A precipitação pluvial média anual dos últimos 30 anos, na área, foi de aproximadamente $1.503 \mathrm{~mm}$ (Silva et al., 2014).

O sistema de manejo avaliado é o ILP tipo Santa Fé, consolidado a partir do ano 2000. A pastagem estudada de Urochloa spp., conduzida em uma área de aproximadamente 7 ha, estava no terceiro ano de formação e foi implantada na época chuvosa, após dois anos e meio de rotação das culturas anuais. A área sob pastagem foi utilizada na recria de bovinos de corte da raça zebuína Nelore BRGN, desenvolvida e melhorada para a região do Cerrado. Os animais pastejavam em curto período de tempo, pois se fazia o rodízio deles nas diferentes áreas do sistema ILP, para aumentar a 
taxa de lotação animal. Os animais foram manejados na área da pastagem, nos seguintes períodos e com as respectivas taxa de lotação animal (UA - unidade animal): 24/1/2013 a 5/2/2013, 15,88 UA ha ${ }^{-1}$; 18/2/2013 a 6/3/2013, 15,88 UA ha-1; 26/3/2013 a 2/4/2013, 15,88 UA ha ${ }^{-1} ; 15 / 4 / 2013$ a 17/4/2013, 15,88 $\mathrm{UAha}^{-1} ; 24 / 5 / 2013$ a 20/7/2013, 7,58 UA ha $^{-1} ; 8 / 8 / 2013$ a 14/8/2013, 14,02 UA ha-1 ${ }^{-1} 14 / 9 / 2013$ a 20/9/2013, $14,02 \mathrm{UA} \mathrm{ha}^{-1}$.

O estudo foi realizado de 5 de fevereiro a 30 de setembro de 2013 e englobou parte da época chuvosa e da seca. Realizaram-se duas adubações nitrogenadas: uma em 11 de março de 2013, com a aplicação de 100 $\mathrm{kg}$ de ureia ha- ${ }^{-1}\left(45 \mathrm{~kg} \mathrm{ha}^{-1}\right.$ de $\left.\mathrm{N}\right)$; e outra em 8 de julho de 2013, com aplicação de 222,22 $\mathrm{kg}$ de ureia ha-1 ${ }^{-1}(100$ $\mathrm{kg} \mathrm{ha}^{-1} \mathrm{de} \mathrm{N}$ ). A ureia foi aplicada a lanço e distribuída em toda a área da pastagem. A última correção do solo com calcário foi realizada no verão de 2002/2003, com a aplicação de $2.000 \mathrm{~kg} \mathrm{ha}^{-1}$; nessa ocasião, na área da pastagem, se iniciou o cultivo de milho sob plantio direto, em associação com Urochloa spp. cultivada como planta de cobertura.

As coletas foram realizadas semanalmente, com exceção dos períodos de adubação e precipitação pluvial, em que foram frequentes até a estabilização desses eventos. No caso da adubação, os fluxos foram amostrados por sete dias consecutivos, após a aplicação da ureia ao solo.

$\mathrm{Na}$ quantificação dos fluxos de $\mathrm{N}_{2} \mathrm{O}$, utilizou-se o método da câmara estática manual. Foram marcados 25 pontos de amostragem na área da pastagem, equidistantes entre si em $25 \mathrm{~m}$, que formaram uma rede de $5 \times 5$ câmaras. As câmaras foram dispostas em uma área de $10.000 \mathrm{~m}^{2}$ dentro da pastagem. $\mathrm{Na}$ área do cerrado nativo, as câmaras estáticas foram instaladas a no mínimo $50 \mathrm{~m}$ da borda da vegetação. Foram utilizadas cinco câmaras, equidistantes entre si em 5 m. A câmara estática utilizada foi do tipo base-tampa, confeccionada a partir de aço galvanizado (40x60x15 $\mathrm{cm}$ de largura, comprimento e altura, respectivamente). A base da câmara foi inserida no solo a $0,10 \mathrm{~m}$ de profundidade, e nivelada conforme a declividade do terreno. As amostragens se concentraram no período da manhã, entre 9 e $10 \mathrm{~h}$, e os tempos de coleta foram de 0,15 e $30 \mathrm{~min}$, após o fechamento das câmaras. Simultaneamente às coletas, as temperaturas do ar e do solo foram monitoradas com termômetro digital portátil. A concentração de $\mathrm{N}_{2} \mathrm{O}$ foi determinada por cromatografia gasosa (Perkin Elmer Auto System XL, PerkinElmer, Norwalk, CT, USA). O cromatógrafo foi equipado com coluna empacotada, que continha Porapak Q e funcionava a $65^{\circ} \mathrm{C}$, e detector de captura de elétrons $63 \mathrm{Ni}(\mathrm{ECD})$ a $375^{\circ} \mathrm{C}$. Para a calibração do cromatógrafo, utilizaram-se padrões primários de $\mathrm{N}_{2} \mathrm{O}$ à concentração de 350 e 1.000 ppbv (parte por bilhão, na base de volume).

As concentrações de $\mathrm{N}_{2} \mathrm{O}$ foram calculadas por meio de regressões lineares. Quando os fluxos eram subestimados na regressão linear, a função de Hutchinson \& Mosier (1981) era utilizada [função $\mathrm{HM}]$, da seguinte forma: $\mathrm{F}=(\mathrm{C} 1-\mathrm{C} 0)^{2} /[\mathrm{t} 1 \times(2 \times$ $\mathrm{C} 1-\mathrm{C} 2-\mathrm{C} 0)] \times \ln [(\mathrm{C} 1-\mathrm{C} 0) /(\mathrm{C} 2-\mathrm{C} 1)]$, em que: $\mathrm{F}$ é o fluxo $\left(\mu \mathrm{L}\right.$ gás $\left.\mathrm{L}^{-1} \mathrm{~h}^{-1}\right)$; $\mathrm{C} 0, \mathrm{C} 1$, e $\mathrm{C} 2$ são as concentrações (ppmb - parte por bilhão, na base de volume) do gás na câmara de medição, nos tempos 0,1 e 2, respectivamente; e t1 é o intervalo entre os tempos de amostragem (h).

As avaliações físicas e químicas do solo foram feitas em amostras deformadas e indeformadas, coletadas da camada de $0-0,10 \mathrm{~m}$, no total de 25 e 5 repetições, nas áreas da pastagem e no cerrado nativo, respectivamente. As amostras indeformadas foram coletadas em anéis volumétricos de Kopeck, com $5 \times 5 \mathrm{~cm}$ de altura e diâmetro, respectivamente, e as amostras deformadas foram coletadas com o uso do trado calador.

Nas amostras indeformadas, foram determinados: densidade do solo (Ds), porosidade total (PT), microporos (MiPs) e macroporos (MaPs). Os MiPs e MaPs foram determinados por meio de mesa de tensão, à 0,06 atm. (Claessen, 1997). Nas amostras deformadas, foram determinados: o teor de nitrato $\left(\mathrm{NO}_{3}^{-}\right)$e de amônio $\left(\mathrm{NH}_{4}^{+}\right)$, a umidade gravimétrica $(\theta \mathrm{g})$, o pH, a matéria orgânica do solo (MOS) e C e $\mathrm{N}$ total $\left(\mathrm{C}_{\mathrm{t}}\right.$ e $\left.\mathrm{N}_{\mathrm{t}}\right)$ do solo. Por meio da $\theta \mathrm{g}$ e da Ds, foi possível calcular a percentagem do EPPA, por meio da equação descrita por Paul \& Clark (1996).

$\mathrm{Na}$ realização das análises químicas de $\mathrm{pH}, \mathrm{MOS}$, $\mathrm{C}_{t}$ e $\mathrm{N}_{t}$, as amostras de solo foram secas ao ar e peneiradas em peneira de malha de $2 \mathrm{~mm}$ (terra fina seca ao ar, TFSA). $\mathrm{O}$ pH foi determinado pelo método potenciométrico, em que o potencial eletrônico é apurado por meio de eletrodo imerso em suspensão aquosa, à proporção sólido: líquido de 1:2,5 (Claessen, 1997). A MOS foi determinada pelo método de Walkley-Black modificado (Claessen, 1997). Os teores de $\mathrm{NO}_{3}{ }^{-}$e $\mathrm{NH}_{4}{ }^{+}$foram determinados em análises 
quantitativas, por espectrometria UV-Vis acoplada a um sistema FIA (Flow Injection Analysis, FIALAB 2500, Fialab Inc., Seattle, Washington, USA). Os resultados da análise foram corrigidos de acordo com $\theta \mathrm{g}$ do solo do momento da coleta, para a obtenção em base de massa de solo seco.

$\mathrm{O} \mathrm{C}_{t}$ e $\mathrm{N}_{\mathrm{t}}$ do solo foram determinados pelo método de combustão via seca, a $925^{\circ} \mathrm{C}$, com o auxílio de um analisador elementar (Perkin Elmer CHNS/O 2400 Série II, PerkinElmer, Shelton, CT, USA). As amostras analisadas foram previamente moídas e passadas em peneira de 60 mesh. Foram pesadas de 10 a $12 \mathrm{mg}$ de solo em cápsulas de estanho.

Análises descritivas (média e erro padrão) dos valores dos fluxos de $\mathrm{N}_{2} \mathrm{O}$ e das variáveis físicas e químicas do solo foram realizadas, e a normalidade dos dados foi testada por meio do teste de Shapiro-Wilk. Com a finalidade de comparar as variáveis físicas e químicas do solo obtidas em cada sistema - pastagem e cerrado nativo -, aplicaram-se os seguintes testes: $\mathrm{t}$, aos dados que apresentaram distribuição normal; e o teste de Wilcoxon para amostras independentes, aos dados que apresentaram distribuição não normal. A correlação de Spearman foi aplicada aos valores de fluxos de $\mathrm{N}_{2} \mathrm{O}$ e das variáveis físicas e químicas do solo. As análises estatísticas foram realizadas por meio dos programas estatísticos Assistat 7.7, Action e SigmaPlot versão 12.0 .

\section{Resultados e Discussão}

Nos dados de fluxos de $\mathrm{N}_{2} \mathrm{O}$ da área sob pastagem e cerrado nativo, verificaram-se valores médios que variaram de negativos, como $-5,86 \pm 0,84$ e $-8,36 \pm 2,34$ ng $\mathrm{m}^{-2} \mathrm{~s}^{1}$ - que indicam consumo de $\mathrm{N}_{2} \mathrm{O}$ atmosférico, conforme Nogueira et al. (2015) -; baixos, como $0,44 \pm 1,67$ e $0,26 \pm 1,28 \mathrm{ng} \mathrm{m}^{-2} \mathrm{~s}^{-1}$, na área sob pastagem e cerrado nativo, respectivamente; e muito elevados, na ordem de $290,34 \pm 43,23 \mathrm{ng} \mathrm{m}^{-2} \mathrm{~s}^{-1}$, na área sob pastagem.

Entre os fatores que influenciaram os fluxos de $\mathrm{N}_{2} \mathrm{O}$, podem ser citados os seguintes: os edáficos, a exemplo da quantidade de MiPs que influenciou o EPPA do solo; a microporosidade que, aliada ao alto teor de água, pode gerar condições redutoras e, assim, ocasionar maior disponibilidade de $\mathrm{N}$ com consequente aumento das emissões de $\mathrm{N}_{2} \mathrm{O}$ dos solos (Drury et al., 2004); o uso de fertilizante nitrogenado e de dejetos animais
(Lima, 2000); o retorno de $\mathrm{N}$ por meio da deposição de urina e esterco, em áreas sob pastejo, que ocorre de forma localizada (Sordi et al., 2014) e proporciona sítios de alta produção de $\mathrm{N}_{2} \mathrm{O}$, já que grande parte do $\mathrm{N}$ consumido pelos animais é excretada (Lessa et al., 2014); e os fatores climáticos, que variam conforme o período do ano.

As precipitações pluviais favorecem o aumento do teor de umidade do solo e, consequentemente, o deficit de oxigênio, condição ideal para a desnitrificação, processo-chave na produção de $\mathrm{N}_{2} \mathrm{O}$ no solo (Davidson et al., 2000). A temperatura do solo afeta a atividade dos microrganismos. $\mathrm{O}$ aumento da temperatura provoca uma intensificação das transformações no solo, principalmente quanto à mineralização do material orgânico, à dinâmica do $\mathrm{N}$ e às emissões dos gases do solo (Haney et al., 2004).

Os fluxos médios de $\mathrm{N}_{2} \mathrm{O}$, na área da pastagem e no cerrado nativo, foram 14,12 e -2,45 $\mathrm{ng} \mathrm{m}^{-2} \mathrm{~s}^{-1}$, respectivamente. A emissão total obtida na área da pastagem foi de $1.644,19 \mathrm{~g} \mathrm{ha}^{-1}$. Na área de cerrado nativo, não houve emissão de $\mathrm{N}_{2} \mathrm{O}$ do solo para a atmosfera. Nogueira et al. (2015) relataram fluxos médios de $\mathrm{N}_{2} \mathrm{O}$ de 4,17 e $1,67 \mathrm{ng} \mathrm{m}^{-2} \mathrm{~s}^{-1}$, em áreas de pastagem manejada e de floresta, respectivamente; além de fluxos médios, em outra localidade, de 17,50 e 1,39 $\mathrm{ng} \mathrm{m}^{-2} \mathrm{~s}^{-1}$, nesses mesmos usos do solo, respectivamente.

Os valores de Ds, PT, MiPs, MaP, EPPA e $\theta \mathrm{g}$ diferiram entre a pastagem e o cerrado nativo (Tabela 1). O solo sob cerrado nativo apresentou menor Ds e melhor distribuição dos valores de MaP e MiP do que o solo sob pastagem do ILP.

Segundo Jorge et al. (2012), os solos do bioma Cerrado são caracterizados por excelentes atributos físicos em condições naturais, o que foi verificado nos resultados encontrados no presente estudo, em que a área sob cerrado nativo apresentou melhor estrutura física do solo. A Ds é um atributo cujo valor varia em razão do manejo adotado no sistema (Jorge et al., 2012) ou conforme o uso de operações mecanizadas, e esse valor é condicionado por diversos fatores como agregação do solo, compactação, porosidade, entre outros (Bicalho, 2011); além disso, podem ocorrer alterações na distribuição e no tamanho dos poros e, consequentemente, a tensão com que a água é retida (Larson \& Gupta, 1980). Isso foi verificado na presente pesquisa, em que a área sob pastagem apresentou menor

Pesq. agropec. bras., Brasília, v.51, n.9, p.1148-1155, set. 2016 DOI: 10.1590/S0100-204X2016000900014 
PT, menor quantidade de MaPs e maior quantidade de MiPs - este último associado à maior percentagem de EPPA do que o da área sob cerrado nativo (Tabela 1).

O conteúdo de água no solo variou com a precipitação pluvial. A $\theta \mathrm{g}$ na camada de $0-0,10 \mathrm{~m}$ variou entre 9,1 e $29,5 \%$, no solo sob pastagem, e entre 16,9 e $34 \%$, no solo sob cerrado nativo. Os valores de $\theta \mathrm{g}$ do solo encontrados na área da pastagem foram inferiores aos da área do cerrado nativo. Esse comportamento está relacionado ao processo de compactação do solo, que promove a redução da macroporosidade e PT.

$\mathrm{O}$ solo sob pastagem apresentou maior teor médio de $\mathrm{NH}_{4}{ }^{+}$. Provavelmente, as adubações nitrogenadas, a deposição de fezes e urina pelos animais e a decomposição da palha da braquiária favoreceram a maior concentração de $\mathrm{NH}_{4}^{+}$no solo. Segundo Subbarão et al. (2013), certas plantas podem suprimir o processo de nitrificação do solo por meio da liberação de inibidores pelas raízes, tal fenômeno é observado em gramíneas. Os estudos de campo realizados por tais autores sugerem, ainda, que gramíneas do gênero Urochloa spp. podem suprimir a nitrificação e a emissão de $\mathrm{N}_{2} \mathrm{O}$ do solo.

Estudos como o de Sidiras \& Pavan (1985) indicam que o sistema plantio direto consolidado demanda

Tabela 1. Média das variáveis físicas e químicas de solo sob pastagem, em sistema de integração lavoura-pecuária, e de solo sob cerrado nativo ${ }^{(1)}$.

\begin{tabular}{lcc}
\hline Variável $^{(2)}$ & Pastagem & Cerrado nativo \\
\hline Densidade do solo $\left(\mathrm{g} \mathrm{cm}^{-3}\right)$ & $1,35^{* * *}$ & 0,96 \\
Porosidade total (\%) & $51,75^{* *}$ & 56,02 \\
Microporos (\%) & $40,97^{* * *}$ & 30,45 \\
Macroporos (\%) & $10,78^{* * *}$ & 25,58 \\
EPPA (\%) & $57,00^{* * *}$ & 34,54 \\
Umidade gravimétrica (\%) & $19,99^{* * *}$ & 22,72 \\
pH & $5,79^{* * *}$ & 4,64 \\
Matéria orgânica do solo & $34,31^{\mathrm{ns}}$ & 35,48 \\
Carbono total $\left(\mathrm{g} \mathrm{kg}^{-1}\right)$ & $22,87^{* * *}$ & 25,22 \\
Nitrogênio total $\left(\mathrm{g} \mathrm{kg}^{-1}\right)$ & $1,55^{* *}$ & 1,67 \\
$\mathrm{Relação}_{\mathrm{t}} / \mathrm{N}_{\mathrm{t}}$ & $14,77^{\mathrm{ns}}$ & 15,09 \\
$\mathrm{NO}_{3}{ }^{-}\left(\mathrm{mg} \mathrm{N}^{-} \mathrm{NO}_{3}{ }^{-} \mathrm{kg}^{-1}\right)$ & $30,68^{* * *}$ & 62,37 \\
$\mathrm{NH}_{4}{ }^{+}\left(\mathrm{mg} \mathrm{N}^{-\mathrm{NH}_{4}}{ }^{+} \mathrm{kg}^{-1}\right)$ & $37,28^{* * *}$ & 10,90 \\
\hline
\end{tabular}

${ }^{(1)}$ Dados referentes às coletas de solo à profundidade $0-0,10 \mathrm{~m}$, entre fevereiro e setembro de 2013. ${ }^{(2)} \mathrm{O}$ teste $\mathrm{t}$ foi aplicado para avaliar as variáveis densidade do solo, macroporos, microporos, porosidade total e carbono total; e o teste de Wilcoxonn, para as variáveis espaço poroso preenchido por água (EPPA), umidade gravimétrica, $\mathrm{pH}$, matéria orgânica do solo, nitrogênio total $\left(\mathrm{N}_{\mathrm{t}}\right)$, relação $\mathrm{C}_{\mathrm{t}} / \mathrm{N}_{\mathrm{t}}$, amônio $\left(\mathrm{NH}_{4}^{+}\right)$e nitrato $\left(\mathrm{NO}_{3}{ }^{-}\right)$. ${ }^{\text {ns }}$ Não significativo. ${ }^{* * *} \mathrm{e}^{* *}$ Significativo a 0,1 e $1,0 \%$ de probabilidade, respectivamente. menores teores de calcário, e que, em contrapartida, o teor de alumínio trocável e a percentagem de saturação da CTC efetiva por alumínio tendem a diminuir com o decorrer dos anos de implantação do sistema. O alto teor de MOS e o aporte de resíduos vegetais e animais mantidos na área de ILP pode ter favorecido o aumento e a manutenção dos níveis de $\mathrm{pH}$ na área sob pastagem, que apresentou maior valor médio do que a área do cerrado nativo.

A relação $C_{t} / N_{t}$ do solo da pastagem foi comparável à do solo do cerrado nativo (Tabela 1). Porém, as perdas de $\mathrm{C}_{\mathrm{t}}$ nos solos sob pastagem e cerrado foram de 7,1 e $9,3 \%$, respectivamente.

No período das avaliações, os fluxos de $\mathrm{N}_{2} \mathrm{O}$ apresentaram correlação positiva e significativa com o teor de $\mathrm{NO}_{3}^{-}$, EPPA e a temperatura do solo (Tabela 2). Embora o $\mathrm{NH}_{4}{ }^{+}$estivesse presente no solo em maiores concentrações, $\mathrm{O} \mathrm{NO}_{3}^{-}$afetou significativamente as emissões de $\mathrm{N}_{2} \mathrm{O}$.

Altas taxas de $\mathrm{N}_{2} \mathrm{O}$ ocorrem quando o solo apresenta EPPA acima de $60 \%$, o que dificulta a difusão de $\mathrm{O}_{2} \mathrm{e}$ favorece a formação de ambientes anaeróbicos ideais para a desnitrificação (Bateman \& Baggs, 2005). $\mathrm{Na}$ maioria das vezes, esse processo é intensificado quando há aumento do teor de $\mathrm{NO}_{3}{ }^{-}$no solo (Carmo et al., 2005; Ruser et al., 2006) e quando fatores como temperatura e $\mathrm{C}$ orgânico disponível não são limitantes (Dalal et al., 2003).

Segundo Chapuis-Lardy et al. (2007), a quantidade de $\mathrm{N}_{2} \mathrm{O}$ a ser emitida será maior quando a disponibilidade de $\mathrm{NO}_{3}{ }^{-}$no solo for elevada, pois esse composto é preferido como aceptor de elétrons em

Tabela 2. Correlação bivariada de Spearman entre os valores médios dos fluxos de $\mathrm{N}_{2} \mathrm{O}$ e das variáveis físicas e químicas do solo, na área sob cultivo de pastagem em sistema de integração lavoura-pecuária, no período de 5 de fevereiro a 30 de setembro de 2013.

\begin{tabular}{lccccccc}
\hline Variável & $\mathrm{N}_{2} \mathrm{O}$ & $\mathrm{pH}$ & $\mathrm{EPPA}$ & $\mathrm{NH}_{4}^{+}$ & $\begin{array}{r}\mathrm{NO}_{3} \\
\mathrm{~N}\end{array}$ & $\begin{array}{r}\mathrm{T} \text { solo } \\
\left({ }^{\circ} \mathrm{C}\right)\end{array}$ & $\begin{array}{r}\mathrm{T} \text { ar } \\
\left({ }^{\circ} \mathrm{C}\right)\end{array}$ \\
\hline $\mathrm{N}{ }_{2} \mathrm{O}$ & & $-0,092$ & 0,538 & 0,404 & 0,716 & 0,397 & 0,034 \\
$\mathrm{EH}$ & $\mathrm{ns}$ & & 0,358 & 0,038 & $-0,382$ & 0,059 & $-0,060$ \\
$\mathrm{EPPA}$ & $* * *$ & $\mathrm{~ns}$ & & $-0,264$ & $-0,292$ & 0,565 & 0,116 \\
$\mathrm{NH}_{4}^{+}$ & $\mathrm{ns}$ & $\mathrm{ns}$ & $\mathrm{ns}$ & & 0,667 & 0,147 & $-0,011$ \\
$\mathrm{NO}_{3}{ }^{-}$ & $* * *$ & $\mathrm{~ns}$ & $\mathrm{~ns}$ & $* * *$ & & 0,211 & $-0,016$ \\
$\mathrm{~T}$ solo $\left({ }^{\circ} \mathrm{C}\right)$ & $* *$ & $\mathrm{~ns}$ & $* * *$ & $\mathrm{~ns}$ & $\mathrm{~ns}$ & & 0,620 \\
$\mathrm{~T}$ ar $\left({ }^{\circ} \mathrm{C}\right)$ & $\mathrm{ns}$ & $\mathrm{ns}$ & $\mathrm{ns}$ & $\mathrm{ns}$ & $\mathrm{ns}$ & $* * *$ & \\
\hline
\end{tabular}

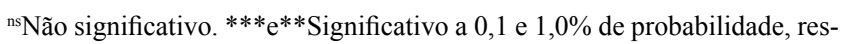
pectivamente. EPPA, espaço poroso preenchido por água. 
relação ao $\mathrm{N}_{2} \mathrm{O}$. A temperatura e a umidade do solo são fatores que influenciam os processos biológicos do solo, pois afetam a atividade dos microrganismos que realizam tais processos. Perdomo et al. (2009) observaram maiores fluxos de $\mathrm{N}_{2} \mathrm{O}$, principalmente após a ocorrência de precipitações pluviais e durante períodos de alta temperatura do solo.

Com o intuito de avaliar o efeito da estação do ano sobre a incidência dos fluxos de $\mathrm{N}_{2} \mathrm{O}$, estes foram correlacionados aos atributos físicos do solo na estação chuvosa e aos atributos físicos e químicos do solo na estação seca. Na estação chuvosa, observou-se correlação positiva entre os fluxos de $\mathrm{N}_{2} \mathrm{O}$ e o EPPA do solo (Tabela 3). As precipitações pluviais favoreceram a mineralização do $\mathrm{N}$ orgânico oriundo das fezes dos animais e a hidrólise da ureia aplicada ao solo e, como consequência, houve aumento dos fluxos de $\mathrm{N}_{2} \mathrm{O}$. $\mathrm{Na}$ estação seca, observou-se correlação positiva e significativa entre os fluxos de $\mathrm{N}_{2} \mathrm{O}$ e os teores de $\mathrm{NH}_{4}{ }^{+}$ e $\mathrm{NO}_{3}{ }^{-}$do solo. A ocorrência das precipitações pluviais, após o período seco, favoreceu as maiores magnitudes de fluxos de $\mathrm{N}_{2} \mathrm{O}$ dentro do período estudado, conforme relatado por Varella et al. (2004). Em períodos secos, os processos de nitrificação e de desnitrificação podem ocorrer de forma simultânea no solo com a ocorrência

Tabela 3. Correlação bivariada de Spearman, entre os valores médios dos fluxos de óxido nitroso e das variáveis físicas e químicas do solo, na área sob cultivo de pastagem em sistema de integração lavoura-pecuária, nos períodos chuvoso, de 5 de fevereiro a 24 de abril de 2013, e seco, de 15 de maio a 30 de setembro de 2013.

\begin{tabular}{lccccccc}
\hline Variável & $\mathrm{N}_{2} \mathrm{O}$ & $\mathrm{pH}$ & $\mathrm{EPPA}$ & $\mathrm{NH}_{4}^{+}$ & $\mathrm{NO}_{3}^{-}$ & $\begin{array}{c}\mathrm{T} \text { solo } \\
\left({ }^{\circ} \mathrm{C}\right)\end{array}$ & $\begin{array}{c}\mathrm{T} \text { ar } \\
\left({ }^{\circ} \mathrm{C}\right)\end{array}$ \\
\hline \multicolumn{7}{c}{ Época chuvosa } \\
$\mathrm{N}_{2} \mathrm{O}$ & & - & 0,671 & - & - & 0,034 & $-0,209$ \\
$\mathrm{EPPA}$ & $* *$ & - & & - & - & $-0,342$ & $-0,322$ \\
$\mathrm{~T}$ solo $\left({ }^{\circ} \mathrm{C}\right)$ & $\mathrm{ns}$ & - & $\mathrm{ns}$ & - & - & & 0,715 \\
$\mathrm{~T}$ ar $\left({ }^{\circ} \mathrm{C}\right)$ & $\mathrm{ns}$ & - & $\mathrm{ns}$ & - & - & $* * *$ & \\
\hline \multicolumn{7}{c}{ Época seca } \\
$\mathrm{N}{ }_{2} \mathrm{O}$ & & 0,041 & 0,176 & 0,466 & 0,788 & 0,207 & $-0,017$ \\
$\mathrm{pH}$ & $\mathrm{ns}$ & & 0,384 & 0,229 & $-0,153$ & $-0,123$ & $-0,265$ \\
$\mathrm{EPPA}$ & $\mathrm{ns}$ & $\mathrm{ns}$ & & 0,135 & $-0,200$ & 0,024 & $-0,072$ \\
$\mathrm{NH}_{4}{ }^{+}$ & $*$ & $\mathrm{~ns}$ & $\mathrm{~ns}$ & & 0,636 & 0,716 & 0,285 \\
$\mathrm{NO}_{3}^{-}$ & $* * *$ & $\mathrm{~ns}$ & $\mathrm{~ns}$ & $* *$ & & 0,705 & 0,256 \\
$\mathrm{~T}$ solo $\left({ }^{\circ} \mathrm{C}\right)$ & $\mathrm{ns}$ & $\mathrm{ns}$ & $\mathrm{ns}$ & $* * *$ & $* * *$ & & 0,568 \\
$\mathrm{~T}$ ar $\left({ }^{\circ} \mathrm{C}\right)$ & $\mathrm{ns}$ & $\mathrm{ns}$ & $\mathrm{ns}$ & $\mathrm{ns}$ & $\mathrm{ns}$ & $* *$ & \\
\hline
\end{tabular}

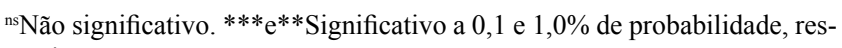
pectivamente. de precipitações pluviais (Souza \& Enrich-Prast, 2012) e favorecer a formação de $\mathrm{N}_{2} \mathrm{O}$. A correlação positiva observada entre os fluxos de $\mathrm{N}_{2} \mathrm{O}$ e os teores de $\mathrm{NH}_{4}{ }^{+}$ e $\mathrm{NO}_{3}{ }^{-}$no solo indica que tais processos ocorreram de forma simultânea e favoreceram a formação de $\mathrm{N}_{2} \mathrm{O}$.

\section{Conclusões}

1. Os fluxos de $\mathrm{N}_{2} \mathrm{O}$ apresentam correlação positiva com o teor de nitrato, o espaço preenchido pela água do solo e a temperatura do solo.

2. Na época chuvosa, os fluxos apresentam correlação positiva com o espaço preenchido pela água do solo e, na época seca, com o teor de amônio e o nitrato do solo.

3. O solo sob pastagem em sistema de integração lavoura-pecuária apresenta fluxos maiores de $\mathrm{N}_{2} \mathrm{O}$, principalmente após longo período seco precedido de precipitações pluviais.

4. $\mathrm{Na}$ área sob Cerrado há ocorrência predominante de fluxos negativos de $\mathrm{N}_{2} \mathrm{O}$.

5. O solo com pastagem manejada em sistema de integração lavoura-pecuária apresenta perdas de $\mathrm{N}_{2} \mathrm{O}$ para a atmosfera, enquanto no cerrado nativo não há emissão de $\mathrm{N}_{2} \mathrm{O}$.

\section{Agradecimentos}

Ao Conselho Nacional de Desenvolvimento Científico e Tecnológico (CNPq, projeto 562601/ 2010-14), à Fundação de Amparo à Pesquisa do Estado de Goiás (Fapeg, 20090044900417) e à Coordenação de Aperfeiçoamento de Pessoal de Nivel Superior (Capes), pelo auxílio financeiro.

\section{Referências}

ALVARENGA, R.C.; NOCE, M.A. Integração lavoura-pecuária. Sete Lagoas: Embrapa Milho e Sorgo, 2005. 16p. (Embrapa Milho e Sorgo. Documentos, 47).

BATEMAN, E.J.; BAGGS, E.M. Contributions of nitrification and denitrification to $\mathrm{N}_{2} \mathrm{O}$ emissions from soils at different water-filled pore space. Biology and Fertility of Soils, v.41, p.379-388, 2005. DOI: $10.1007 / \mathrm{s} 00374-005-0858-3$.

BICALHO, I.M. Um estudo da densidade do solo em diferentes sistemas de uso e manejo. Enciclopédia Biosfera, v.7, p.1-9, 2011. BOUWMAN, A.F.; BOUMANS, L.J.M.; BATJES, N.H. Modeling global annual $\mathrm{N}_{2} \mathrm{O}$ and $\mathrm{NO}$ emissions from fertilized fields. Global Biogeochemical Cycles, v.16, p.28-1-28-9, 2002. DOI: $10.1029 / 2001 \mathrm{gb} 001812$.

Pesq. agropec. bras., Brasília, v.51, n.9, p.1148-1155, set. 2016 DOI: 10.1590/S0100-204X2016000900014 
BRASSEUR, G.P.; ORLANDO, J.J.; TYNDALL, G.S. Atmospheric chemistry and global change. New York: Oxford University, 1999. 654p.

CARMO, J.B. do; ANDRADE, C.A. de; CERRI, C.C.; PICCOLO, M. de C. Disponibilidade de nitrogênio e fluxos de $\mathrm{N}_{2} \mathrm{O}$ a partir de solo sob pastagem após aplicação de herbicida. Revista Brasileira de Ciência do Solo, v.29, p.843-852, 2005. DOI: 10.1590/S010006832005000500009.

CERRI, C.C.; CERRI, C.E.P. Agricultura e aquecimento global. Boletim da Sociedade Brasileira de Ciência do Solo, v.32, p.4044, 2007.

CHAPUIS-LARDY, L.; WRAGE, N.; METAY, A.; CHOTTE, J.-L.; BERNOUX, M. Soils, a sink for $\mathrm{N}_{2} \mathrm{O}$ ? A review. Global Change Biology, v.13, p.1-17, 2007. DOI: 10.1111/j.13652486.2006.01280.x.

CLAESSEN, M.E.C. (Org.). Manual de métodos de análise de solo. 2.ed. rev. atual. Rio de Janeiro: Embrapa-CNPS, 1997. 212p. (Embrapa-CNPS. Documentos, 1).

DALAL, R.C.; WANG, W.; ROBERTSON, G.P.; PARTON, W.J. Nitrous oxide emission from Australian agricultural lands and mitigation options: a review. Australian Journal of Soil Research, v.41, p.165-195, 2003. DOI: 10.1071/SR02064.

DAVIDSON, E.A.; KELLER, M.; ERICKSON, H.E.; VERCHOT, L.V.; VELDKAMP, E. Testing a conceptual model of soil emissions of nitrous and nitric oxides. BioScience, v.50, p.667-680, 2000. DOI: 10.1641/0006-3568(2000)050[0667:TAC.

DRURY, C.F.; YANG, X.M.; REYNOLDS, W.D.; TAN, C.S. Influence of crop rotation and aggregate size on carbon dioxide production and denitrification. Soil and Tillage Research, v.79, p.87-100, 2004. DOI: 10.1016/j.still.2004.03.020.

ESTIMATIVAS anuais de emissões de gases de efeito estufa no Brasil. Brasília: Ministério da Ciência e Tecnologia, 2013. 76p.

GIACOMINI, S.J.; JANTALIA, C.P.; AITA, C.; URQUIAGA, S.S.; ALVES, B.J.R. Emissão de óxido nitroso com a aplicação de dejetos líquidos de suínos em solo sob plantio direto. Pesquisa Agropecuária Brasileira, v.41, p.1653-1661, 2006. DOI: 10.1590/ S0100-204X2006001100012.

HANEY, R.L.; FRANZLUEBBERS, A.J.; PORTER, E.B.; HONS, F.M.; ZUBERER, D.A. Soil carbon and nitrogen mineralization: influence of drying temperature. Soil Science Society of America Journal, v.68, p.489-492, 2004. DOI: 10.2136/sssaj2004.0489.

HUTCHINSON, G.L.; MOSIER, A.R. Improved soil cover method for field measurement of nitrous oxide fluxes. Soil Science Society of America Journal, v.45, p.311-316, 1981. DOI: 10.2136/ sssaj1981.03615995004500020017x.

JORGE, R.F.; ALMEIDA, C.X. de; BORGES, E.N.; PASSOS, R.R. Distribuição de poros e densidade de Latossolos submetidos a diferentes sistemas de uso e manejo. Bioscience Journal, v.28, p.159-169, 2012.

LAL, R.; FOLLETT, R.F.; STEWART, B.A.; KIMBLE, J.M. Soil carbon sequestration to mitigate climate change and advance food security. Soil Science, v.172, p.943-956, 2007. DOI: 10.1097/ ss.0b013e $31815 \mathrm{cc} 498$.
LARSON, W.E.; GUPTA, S.C. Estimating critical stress in unsaturated soils from changes in pore water pressure during confined compression. Soil Science Society of America Journal, v.44, p.1127-1132, 1980. DOI: 10.2136/ sssaj1980.03615995004400060001x.

LESSA, A.C.R.; MADARI, B.E.; PAREDES, D.S.; BODDEY, R.M.; URQUIAGA, S.; JANTALIA, C.P.; ALVES, B.J.R. Bovine urine and dung deposited on Brazilian savannah pastures contribute differently to direct and indirect soil nitrous oxide emissions. Agriculture, Ecosystems and Environment, v.190, p.104-111, 2014. DOI: 10.1016/j.agee.2014.01.010.

LIMA, M.A. de. Emissão de gases de efeito estufa provenientes de sistemas agrícolas no Brasil. Biotecnologia, Ciência e Desenvolvimento, v.3, p.38-43, 2000.

MACHADO, P.L.O. de A. Carbono do solo e a mitigação da mudança climática global. Química Nova, v.28, p.329-334, 2005. DOI: $10.1590 /$ S0100-40422005000200026.

NOGUEIRA, A.K. da S.; RODRIGUES, R. de A.R.; CASTRO, B.S.; NOGUEIRA, T.F.; SILVA, J.J.N. da; BEHLING, M.; MOMBACH, M.; ARMACOLO, N.; SILVEIRA, J.G. Emissões de óxido nitroso e metano do solo em áreas de recuperação de pastagens na Amazônia matogrossense. Química Nova, v.38, p.937-943, 2015. DOI: 10.5935/0100-4042.20150109.

PAUL, E.A.; CLARK, F.E. Soil microbiology and biochemistry. $2^{\text {nd }}$ ed. San Diego: Academic Press, 1996. 340p.

PERDOMO, C.; IRISARRI, P.; ERNST, O. Nitrous oxide emissions from an Uruguayan Argiudoll under different tillage and rotation treatments. Nutrient Cycling in Agroecosystems, v.84, p.119-128, 2009. DOI: 10.1007/s10705-008-9231-x.

RUSER, R.; FLESSA, H.; RUSSOW, R.; SCHMIDT, G.; BUEGGER, F.; MUNCH, J.C. Emission of $\mathrm{N}_{2} \mathrm{O}, \mathrm{N}_{2}$ and $\mathrm{CO}_{2}$ from soil fertilized with nitrate: effect of compaction, soil moisture and rewetting. Soil Biology and Biochemistry, v.38, p.263-274, 2006. DOI: 10.1016/j.soilbio.2005.05.005.

SANTOS, G.G.; SILVEIRA, P.M. da; MARCHÃO, R.L.; BECQUER, T.; RIOS, A.J.W.; RODRIGUES, C. Descrição morfológica de perfis de solos da Fazenda Capivara da Embrapa Arroz e Feijão: relatório do ano de 2010. Santo Antônio: Embrapa Arroz e Feijão, 2010. 52p. (Embrapa Arroz e Feijão. Documentos, 258).

SIDIRAS, N.; PAVAN, M.A. Influência do sistema de manejo do solo no seu nível de fertilidade. Revista Brasileira de Ciência do Solo, v.9, p.249-254, 1985.

SILVA, S.C. da; HEINEMANN, A.B.; PAZ, R.L.F. da; AMORIM, $A$. de $O$. Informações meteorológicas para pesquisa e planejamento agrícola, referentes ao Município de Santo Antônio de Goiás, GO, 2012. Santo Antônio de Goiás: Embrapa Arroz e Feijão, 2014. 29p. (Embrapa Arroz e Feijão. Documentos, 298).

SNYDER, C.S.; BRUULSEMA, T.W.; JENSEN, T.L.; FIXAN, P.E. Review of greenhouse gas emissions from crop production systems and fertilizer management effects. Agriculture, Ecosystems and Environment, v.133, p.247-266, 2009. DOI: 10.1016/j.agee.2009.04.021. 
SORDI, A.; DIECKOW, J.; BAYER, C.; ALBUQUERQUE, M.A.; PIVA, J.T.; ZANATTA, J.A.; TOMAZI, M.; ROSA, C.M. da; MORAES, A. de. Nitrous oxide emission factors for urine and dung patches in a subtropical Brazilian pastureland. Agriculture Ecosystems and Environment, v.190, p.94-103, 2014. DOI: 10.1016/j.agee.2013.09.004.

SOUZA, V.F.; ENRICH-PRAST, A. Óxido nitroso $\left(\mathrm{N}_{2} \mathrm{O}\right)$ em ambientes aquáticos continentais: produção, fatores reguladores e fluxos de diferentes ambientes. Oecologia Australis, v.16, p.311329, 2012. DOI: 10.4257/oeco.2012.1602.09.

SUBBARÃO, G.V.; RAO, I.M.; NAKAHARA, K.; ANDO, Y.; SAHRAWAT, K.L.; TESFAMARIAM, T.; LATA, J.C.; BOUDSOCQ, S.; MILES, J.W.; ISHITANI, M.; PETERS, M.
Nitrogen management in grasslands and forage-based production systems - role of biological nitrification inhibition (BNI). Tropical Grasslands-Forrajes Tropicales, v.1, p.168-174, 2013. DOI: 10.17138/TGFT(1)168-174.

VARELLA, R.F.; BUSTAMANTE, M.M.C.; PINTO, A.S.; KISSELLE, K.W.; SANTOS, R.V.; BURKE, R.A.; ZEPP, R.G.; VIANA, L.T. Soil fluxes of $\mathrm{CO}_{2}, \mathrm{CO}, \mathrm{NO}$, and $\mathrm{N}_{2} \mathrm{O}$ from an old pasture and from native savanna in Brazil. Ecological Applications, v.14, p.221-231, 2004. DOI: 10.1890/01-6014.

VILELA, L.; MARTHA-JÚNIOR, G.B.; MARCHÃO, R.L. Integração lavoura-pecuária-floresta: alternativa para intensificação do uso da terra. Revista UFG, ano 8, p.92-99, 2012.

Recebido em 31 de agosto de 2015 e aprovado em 11 de fevereiro de 2016 